\title{
Optimization of SPECT/CT imaging protocols for quantitative and qualitative ${ }^{99 \mathrm{~m}} \mathrm{Tc}$ SPECT
}

Dennis Kupitz ${ }^{1 *}$ D, Heiko Wissel ${ }^{1}$, Jan Wuestemann ${ }^{1}$, Stephanie Bluemel ${ }^{2}$, Maciej Pech ${ }^{1,3}$, Holger Amthauer $^{2}$, Michael C. Kreissl ${ }^{1,3}$ and Oliver S. Grosser ${ }^{1,3}$

* Correspondence: dennis.kupitz@ med.ovgu.de

${ }^{1}$ Department of Radiology and Nuclear Medicine, University Hospital Magdeburg, Magdeburg, Germany

Full list of author information is available at the end of the article

\begin{abstract}
Background: The introduction of hybrid SPECT/CT devices enables quantitative imaging in SPECT, providing a methodological setup for quantitation using SPECT tracers comparable to PET/CT. We evaluated a specific quantitative reconstruction algorithm for SPECT data using a ${ }^{99 m}$ Tc-filled NEMA phantom. Quantitative and qualitative image parameters were evaluated for different parametrizations of the acquisition and reconstruction protocol to identify an optimized quantitative protocol.

Results: The reconstructed activity concentration $\left(A C_{\text {rec }}\right)$ and the signal-to-noise ratio (SNR) of all examined protocols ( $n=16$ ) were significantly affected by the parametrization of the weighting factor $k$ used in scatter correction, the total number of iterations and the sphere volume (all, $p<0.0001$ ). The two examined SPECT acquisition protocols (with 60 or 120 projections) had a minor impact on the $A C_{r e c}$ and no significant impact on the SNR.

In comparison to the known AC, the use of default scatter correction ( $k=0.47)$ or objectspecific scatter correction $(k=0.18)$ resulted in an underestimation of $A C_{\text {rec }}$ in the largest sphere volume $(26.5 \mathrm{ml})$ by $-13.9 \mathrm{kBq} / \mathrm{ml}(-16.3 \%)$ and $-7.1 \mathrm{kBq} / \mathrm{ml}(-8.4 \%)$, respectively. An increase in total iterations leads to an increase in estimated $A C$ and a decrease in SNR. The mean difference between $A C_{\text {rec }}$ and known $A C$ decreased with an increasing number of total iterations (e.g., for 20 iterations ( 2 iterations $/ 10$ subsets) $=-14.6$ $\mathrm{kBq} / \mathrm{ml}(-17.1 \%), 240$ iterations $(24 \mathrm{i} / 10 \mathrm{~s})=-8.0 \mathrm{kBq} / \mathrm{ml}(-9.4 \%), p<0.0001)$. In parallel, the mean SNR decreased significantly from $2 \mathrm{i} / 10$ s to $24 \mathrm{i} / 10$ s by $76 \%(p<0.0001)$.

Conclusion: Quantitative SPECT imaging is feasible with the used reconstruction algorithm and hybrid SPECT/CT, and its consistent implementation in diagnostics may provide perspectives for quantification in routine clinical practice (e.g., assessment of bone metabolism). When combining quantitative analysis and diagnostic imaging, we recommend using two different reconstruction protocols with task-specific optimized setups (quantitative vs. qualitative reconstruction). Furthermore, individual scatter correction significantly improves both quantitative and qualitative results.
\end{abstract}

Keywords: Quantitative SPECT, SPECT/CT, Optimization, Scatter correction, Image reconstruction
(-) The Author(s). 2021 Open Access This article is licensed under a Creative Commons Attribution 4.0 International License, which permits use, sharing, adaptation, distribution and reproduction in any medium or format, as long as you give appropriate credit to the original author(s) and the source, provide a link to the Creative Commons licence, and indicate if changes were made. The images or other third party material in this article are included in the article's Creative Commons licence, unless indicated otherwise in a credit line to the material. If material is not included in the article's Creative Commons licence and your intended use is not permitted by statutory regulation or exceeds the permitted use, you will need to obtain permission directly from the copyright holder. To view a copy of this licence, visit http://creativecommons.org/licenses/by/4.0/. 


\section{Background}

During the last decade, quantitative imaging has become a standard in nuclear medicine diagnostics. This benchmark was exemplified by the long-term process in developing PET/CT (positron emission tomography with X-ray computed tomography), representing the current standard in quantitative hybrid imaging $[1,2]$.

In parallel, the introduction of hybrid SPECT/CT (single photon emission computed tomography with computed X-ray tomography) imaging devices provides opportunities for quantitation in SPECT imaging [3]. In this context, quantitative SPECT (qSPECT) provides the possibility to use gamma-emitting radiotracers within a similar setup as PET/CT applications. Apart from superior resolution in PET, this development has to overcome the substantial difference in quantitation between PET/CT and SPECT/CT. Currently, novel clinical applications (e.g., dosimetry in peptide receptor radionuclide therapy) further emphasize the need for the development of qSPECT imaging [4-6].

As a result of improvements in SPECT image reconstruction, dedicated corrections (e.g., attenuation correction and scatter correction) have provided the potential for qSPECT imaging [4, 7-13]. Currently, state-of-the-art attenuation correction in SPECT reconstruction is performed using CT data from hybrid SPECT/CT imaging [13, 14]. Various methods have been implemented for scatter correction [15]. The most commonly used method is based on the parallel acquisition of at least one additional energy window in correlation with the photopeak window (or to each photopeak window, if applicable) [16-19].

In this study, we evaluated a manufacturer-specific quantitative reconstruction algorithm for SPECT imaging using ${ }^{99 \mathrm{~m}} \mathrm{Tc}$ (Technetium-99m)-labelled radiopharmaceuticals. We assessed the accuracy in quantitation of activity concentrations for different parametrizations of the acquisition and reconstruction protocol (e.g., iterations and scatter weighting) to identify an optimized quantitative protocol. In parallel, qualitative image parameters (e.g., signal-to-noise ratio) were measured and evaluated. The phantom setup used for evaluation was adapted to clinical conditions (e.g., observed activity concentrations).

\section{Methods}

\section{Phantom}

Phantom measurements were performed using the standardized NEMA IEC body phantom (Data Spectrum Corporation, Durham, NC, USA) with six fillable spheres (diameters of 10,13, 17, 22, 28 and $37 \mathrm{~mm}$ ) and a cylindrical polystyrene-filled lung insert [20]. The phantom was filled with ${ }^{99 \mathrm{~m}} \mathrm{Tc}$-pertechnetate diluted in water, with specific activities of $80 \mathrm{kBq} / \mathrm{ml}$ for each of the six spheres and $10 \mathrm{kBq} / \mathrm{ml}$ for the background (ratio 8:1). The lung insert, in the centre of the phantom, was not filled with radioactive material.

\section{SPECT/CT: imaging and reconstruction}

Imaging was performed with hybrid SPECT/CT (Discovery NM/CT 670, GE Healthcare, Haifa, Israel) with dual-head $\mathrm{NaI}$ detectors equipped with low-energy, highresolution (LEHR) collimators. SPECT data were acquired using two different acquisition protocols: (1.) a clinical ${ }^{99 \mathrm{~m}} \mathrm{Tc}$ imaging protocol with 60 projections per $360^{\circ}$ (30 
projections per detector) at steps of $6^{\circ}(20 \mathrm{~s} /$ projection) and (2.) a NEMA-oriented imaging protocol [21] with 120 projections per $360^{\circ}$ (60 per detector) at steps of $3^{\circ}(10 \mathrm{~s} /$ projection to achieve identic scan time and comparable total count statistics as setup number 1). The second protocol was chosen to analyse the effect by number of projections for equivalent total examination time. All other imaging parameters (energy window: photopeak $=140.5 \mathrm{keV} \pm 10 \%$, scatter window $=120.0 \mathrm{keV} \pm 5 \%$; image matrix $256 \times 256$, pixel size $=2.21 \times 2.21 \mathrm{~mm}$, and zoom $=1.0$ ) were not changed.

CT imaging was performed using a low-dose protocol $\left(I=40 \mathrm{~mA}, U=120 \mathrm{kVp}, t_{\mathrm{rot}}=0.5\right.$ $\mathrm{s}$, primary collimation $=16 \times 1.25 \mathrm{~mm}$, and pitch $=1.375)$. CT data were reconstructed with a matrix of $512 \times 512$ (pixel size $=0.98 \times 0.98 \mathrm{~mm}$ ) and a slice thickness of $3.75 \mathrm{~mm}$ by filtered back projection (with manufacturer-specific convolution kernel "standard"). The lowdose CT data were used for attenuation correction of the corresponding SPECT data.

Quantitative SPECT reconstruction was performed using a dedicated software module (Q. Metrix, GE Healthcare, Haifa, Israel) [22] used an iterative ordered-subset expectation maximization algorithm (2D OSEM) for image reconstruction with four different parametrizations: (1.) 2 iterations and 10 subsets (2i/10s), (2.) 4 iterations and 10 subsets (4i/10s), (3.) 5 iterations and 15 subsets (5i/15s) and (4.) 24 iterations and 10 subsets $(24 \mathrm{i} / 10 \mathrm{~s})$. The reconstruction algorithm performed corrections for resolution recovery (by using a collimator- and nuclide-specific 3D-point spread function), attenuation and scatter. The dual-energy window method was used for scatter correction [10]. SPECT data were reconstructed in general without postfiltering. For comparison, SPECT data reconstructions with 2i/10s were performed with postfiltering (Butterworth with cut-off frequency $=0.5 \mathrm{cycles} / \mathrm{cm}$ and power $=10$ ) to illustrate the image quality of our local clinical standard for diagnostic ${ }^{99 \mathrm{~m}} \mathrm{Tc}$ imaging.

Analysis was performed for two different scatter weighting factors (SCFs): SCF $=1.10$ $\left(\mathrm{SCF}_{1.10}\right.$, default for ${ }^{99 \mathrm{~m}} \mathrm{Tc}$, recommended by the manufacturer $)$ and $\mathrm{SCF}=0.41\left(\mathrm{SCF}_{0.41}\right.$, optimized for chosen phantom geometry). The optimized $\mathrm{SCF}_{0.41}$ was previously determined in concordance with de Nijs et al. [23] (see supplementary information). For the used energy window widths, the two SCFs can be converted into the more common scatter multipliers (introduced by Jaszczak [16]) $k=0.470$ and 0.175 for $\mathrm{SCF}_{1.10}$ and $\mathrm{SCF}_{0.41}$, respectively [10, 24]. Table 1 shows an overview of all analysed reconstructions.

The conversion from reconstructed counts into activity concentration (qSPECT data in units of $\mathrm{MBq} / \mathrm{ml}$ ) was performed by multiplication with a quantitative factor $\mathrm{QF}$ $(\mathrm{MBq} / \mathrm{cnt} / \mathrm{ml})[22,25]$ :

$$
\mathrm{QF}=\frac{1}{S \cdot T \cdot V_{\text {Voxel }}}
$$

The effective scanning time of the acquisition was represented by $T(T=1200 \mathrm{~s}$, constant for all examined acquisition protocols). $V_{\text {voxel }}$ represents the volume of a single reconstructed voxel $\left(V_{\text {voxel }}=0.01079 \mathrm{ml}\right)$. The planar system sensitivity $S$ of the gamma camera (units of $\mathrm{cnt} / \mathrm{s} / \mathrm{MBq}$ ) was determined in concordance with the NEMA NU 12018 formalism [21].

\section{Volume of interest analysis}

The software PMOD (PMOD Ver. 3.805, PMOD Technologies LLC, Zurich, Switzerland) was used for segmentation of spherical volumes of interest (VOIs) in the 
Table 1 Examined reconstruction protocols $(n=16)$

\begin{tabular}{lll}
\hline & Iterations and subsets & SCF \\
\hline Quantitative reconstructions $^{\mathrm{a}}$ & & \\
Clinical acquisition protocol $^{\mathrm{b}}$ & $4 \mathrm{i} / 10 \mathrm{~s}$ & 0.41 and 1.10 \\
& $5 \mathrm{i} / 15 \mathrm{~s}$ & 0.41 and 1.10 \\
NEMA acquisition protocol $^{c}$ & $24 \mathrm{i} / 10 \mathrm{~s}$ & 0.41 and 1.10 \\
& $4 \mathrm{i} / 10 \mathrm{~s}$ & 0.41 and 1.10 \\
& $5 \mathrm{i} / 15 \mathrm{~s}$ & 0.41 and 1.10 \\
Diagnostic reconstructions $^{\mathrm{d}}$ & $24 \mathrm{i} / 10 \mathrm{~s}$ & 0.41 and 1.10 \\
Clinical acquisition protocol $^{\mathrm{b}}$ & & \\
NEMA acquisition protocol $^{c}$ & $2 \mathrm{i} / 10 \mathrm{~s}^{\mathrm{e}}$ & 0.41 and $1.10^{\mathrm{e}}$ \\
\hline
\end{tabular}

${ }^{\mathrm{a}}$ Without postfiltering,

${ }^{\mathrm{b}}$ Sixty projections with $20 \mathrm{~s} /$ projection,

'One hundred twenty projections with $10 \mathrm{~s} /$ projection,

${ }^{d}$ With postfiltering (Butterworth, cut-off frequency $=0.5 \mathrm{cycles} / \mathrm{cm}$, power $=10$ )

${ }^{\mathrm{e}}$ Institutional standard for diagnostic imaging, $2 \mathrm{i} / 10 \mathrm{~s}$ and $\mathrm{SCF}=1.10$

SCF scatter weighting factor

low-dose CT data by specifying the known diameter of the spheres. Subsequently, these VOIs were transferred to the co-registered reconstructed SPECT. VOIs were defined for all six spheres of the IEC phantom and for a spherical background VOI ( $V=49.9$ $\mathrm{ml})$. For the evaluation of the reconstructed voxel values per sphere, the reconstructed counts were converted into AC values by using equation (1). The AC values of all voxels per sphere were considered in the analysis. The smallest spherical VOI $(V=0.5 \mathrm{ml})$ consists of $n=46$ voxels and the largest spherical VOI $(V=26.5 \mathrm{ml})$ of $n=2438$ voxels. The background VOI contains $n=4612$ voxels.

Recovery coefficients (hot spot recovery coefficients (HSRC)) [26] were determined using the mean $\mathrm{AC}\left(\mathrm{AC}_{\mathrm{rec}}\right)$ in the spheres estimated from the $\mathrm{qSPECT}$ data $\left(\mathrm{AC}_{\text {rec.- }}\right.$ sphere $)$ and the known activity concentration $\left(\mathrm{AC}_{\text {real.sphere }}\right)$ :

$$
\mathrm{HSRC}=\frac{\mathrm{AC}_{\text {rec.sphere }}}{\mathrm{AC}_{\text {real.sphere }}}
$$

The signal-to-noise ratio (SNR) was calculated as the difference of the means of the reconstructed $\mathrm{AC}$ in the sphere and the background in relation to the standard deviation $(\mathrm{SD})$ of the background VOI $\mathrm{SD}_{\text {rec. BG }}[27]$ :

$$
\mathrm{SNR}=\frac{\left(\mathrm{AC}_{\text {rec.sphere }}-\mathrm{AC} \mathrm{C}_{\text {rec.BG }}\right)}{\mathrm{SD}_{\text {rec. } \mathrm{BG}}}
$$

The SPECT image noise $(N)$ was determined within the background by the ratio of $\mathrm{SD}$ of the background VOI and the mean reconstructed AC of the background [27]:

$$
N[\%]=\frac{\mathrm{SD}_{\text {rec. } . \mathrm{BG}}}{\mathrm{AC}_{\text {rec.BG }}} \cdot 100 \%
$$

\section{Statistics}

The R software package (version 3.3.3, R Foundation for Statistical Computing, Vienna, Austria) was used for statistical evaluations of the reconstructed AC values. Levene's 
test and the Shapiro-Wilk test were used to check the equality of variances and normal distribution of the variables, respectively. Differences in nonparametric, dependent variables were tested for significance by using the Friedman test or Wilcoxon signed-rank test, if applicable. ANOVA or the $t$ test with Bonferroni-Holm correction applied for multiple comparisons was used for parametrically distributed dependent variables. All tests were performed two-sided, and significance was assumed at a $p$ value $<0.05$.

\section{Results}

The planar sensitivities for ${ }^{99 \mathrm{~m}} \mathrm{Tc}$ were estimated for detector $1 \mathrm{~S}_{\mathrm{DET} 1}$ and detector 2 $\mathrm{S}_{\mathrm{DET} 2}$ to be $71.7 \mathrm{cps} / \mathrm{MBq}$ and $72.8 \mathrm{cps} / \mathrm{MBq}$, respectively. For methodological limitations, a mean system sensitivity of $S_{\text {system }}=72.3 \mathrm{cps} / \mathrm{MBq}$ was used. The known AC in the spheres was $85.1 \mathrm{kBq} / \mathrm{ml}$ and $10.6 \mathrm{kBq} / \mathrm{ml}$ in the background (ratio $\approx 8: 1$ ).

$\mathrm{AC}_{\mathrm{rec}}, \mathrm{HSRC}$ and SNR were not normally distributed when comparing the analysed structures (VOIs) in the phantom for each examined reconstruction protocol (Table 1). In contrast, $\mathrm{AC}_{\mathrm{rec}}$, HSRC, SNR and Noise were normally distributed for each VOI (e.g., comparing only the largest sphere VOIs or only the background VOIs).

\section{Quantitative results}

\section{Spheres}

The $\mathrm{AC}_{\mathrm{rec}}$ and HSRCs were significantly affected by the sphere volume and by the reconstruction protocol (all $p<0.0001$, result by the Friedman test).

The HSRC depended significantly on sphere volume $(p<0.0001)$. The effect by volume will be exemplified for the two largest spheres: the HSRC of the second largest sphere $(V=11.5 \mathrm{ml}, \mathrm{HSRC}=0.85)$ was significantly reduced by -0.03 (representing $\left.\Delta \mathrm{AC}_{\text {rec }}=-2.7 \mathrm{kBq} / \mathrm{ml}, p=0.01\right)$ compared with the largest sphere $(V=26.5 \mathrm{ml}$, HSRC $=0.88$ ).

The results from ANOVA analysis showed significant dependencies of the HSRC and the $\mathrm{AC}_{\mathrm{rec}}$ on the number of total iterations (all, $p<0.0001$ ), SCF (all, $p<0.0001$ ) and acquisition protocol (all, $p<0.02$ ) for each of the four largest examined sphere volumes (up to $V=2.6 \mathrm{ml}$ ). Table 2 shows the results of an ANOVA post hoc test for the largest examined sphere. The results of the remaining sphere volumes are provided in the supplementary data (supplementary Table 1).

The effect of the number of total iterations and the sphere volume on the HSRC is exemplified for the clinical acquisition protocol with $\mathrm{SCF}_{1.10}$ (manufacturer parametrization) in Fig. 1a. The HSRC curves of the four sets of iterations were significantly different from each other $(p=0.0007)$, and there was an increase in the HSRCs with increasing VOI volumes and with an increasing number of iterations. For example, the median HSRC increased in the specific setup by $0.68\left(\Delta \mathrm{AC}_{\text {rec }}=57.5 \mathrm{kBq} / \mathrm{ml}\right)$ when comparing the smallest sphere $\left(V=0.5 \mathrm{ml}, \mathrm{AC}_{\mathrm{rec}}=14.8 \mathrm{kBq} / \mathrm{ml}\right)$ and the largest sphere $\left(V=26.5 \mathrm{ml}, \mathrm{AC}_{\mathrm{rec}}=72.3 \mathrm{kBq} / \mathrm{ml}\right)$. Increasing the total number of iterations from $2 \mathrm{i} /$ $10 \mathrm{~s}$ to $4 \mathrm{i} / 10 \mathrm{~s}$ showed a significant increase in median HSRCs by 0.06 over all spheres $\left(\Delta \mathrm{AC}_{\mathrm{rec}}=4.9 \mathrm{kBq} / \mathrm{ml}, p=0.03\right)$. Furthermore, median HSRC increased significantly from $2 \mathrm{i} / 10 \mathrm{~s}$ to $5 \mathrm{i} / 15 \mathrm{~s}$ and from $2 \mathrm{i} / 10 \mathrm{~s}$ to $24 \mathrm{i} / 10 \mathrm{~s}$ by $0.10\left(\Delta \mathrm{AC}_{\text {rec }}=8.9 \mathrm{kBq} / \mathrm{ml}, p=\right.$ $0.03)$ and $0.13\left(\Delta \mathrm{AC}_{\mathrm{rec}}=11.2 \mathrm{kBq} / \mathrm{ml}, p=0.03\right)$, respectively. 
Table 2 Effect of examined parameters on $A C_{r e c}$ HSRC and SNR. The results are exemplified for the largest sphere volume in comparison to a reference protocol ${ }^{\mathrm{a}}$.

\begin{tabular}{|c|c|c|c|c|c|c|}
\hline $\begin{array}{l}\text { Parameter of } \\
\text { variation }\end{array}$ & $\begin{array}{l}\Delta \mathrm{AC} \text { rec } \\
(\mathrm{kBq} / \mathrm{ml})\end{array}$ & $p^{\mathbf{b}}$ & $\Delta \mathrm{HSRC}$ & $p^{b}$ & $\Delta$ SNR & $p^{\mathrm{b}}$ \\
\hline SCF: 0.41 & +6.7 & $<0.0001$ & +0.08 & $<0.0001$ & +1.1 & n.s. ${ }^{d}$ \\
\hline Iteration set: $4 \mathrm{i} / 10 \mathrm{~s}$ & +4.1 & $<0.0001$ & +0.05 & $<0.0001$ & -8.2 & $<0.0001$ \\
\hline Iteration set: $5 \mathrm{i} / 15 \mathrm{~s}$ & +5.6 & $<0.0001$ & +0.07 & $<0.0001$ & -11.9 & $<0.0001$ \\
\hline Iteration set: $24 \mathrm{i} / 10 \mathrm{~s}$ & +6.6 & $<0.0001$ & +0.08 & $<0.0001$ & -17.9 & $<0.0001$ \\
\hline Acquisition: NEMAC & -0.9 & 0.04 & -0.01 & 0.04 & +0.6 & n.s. ${ }^{d}$ \\
\hline
\end{tabular}

Analysis performed for the largest sphere. For residual spheres see supplementary Table 1.

${ }^{a}$ Clinical standard for diagnostic imaging and reconstruction (60 projections with $20 \mathrm{~s} /$ projection, $\mathrm{SCF}_{1.10}, 2 \mathrm{i} / 10 \mathrm{~s}$, Butterworth postfilter)

${ }^{\mathrm{b}}$ ANOVA post hoc test

${ }^{\mathrm{C}} \mathrm{NEMA}$ acquisition protocol (120 projections and $10 \mathrm{~s} /$ projection)

${ }^{\mathrm{d}}$ Not significant, tested by ANOVA

$S C F$ scatter weighting factor, $A C_{\text {rec }}$ mean reconstructed activity concentration, $H S R C$ hot spot recovery coefficient, SNR signal-to-noise ratio

For a fixed set of total iterations (e.g., 24i/10s), the median HSRCs of the spheres increase for reconstructions with $\mathrm{SCF}_{0.41}$ in comparison to using a $\mathrm{SCF}_{1.10}$ significantly by $0.07\left(\Delta \mathrm{AC}_{\mathrm{rec}}=5.9 \mathrm{kBq} / \mathrm{ml}\right)$ for the clinical and by $0.06\left(\Delta \mathrm{AC}_{\mathrm{rec}}=5.4 \mathrm{kBq} / \mathrm{ml}\right)$ for the NEMA (both, $p=0.03$ ) acquisition protocol, respectively. Using the clinical acquisition protocol, the median HSRCs of the spheres increased slightly by $0.02(\triangle \mathrm{AC}$ rec $=$ $1.5 \mathrm{kBq} / \mathrm{ml}, p=0.84)$ and by $0.02\left(\Delta \mathrm{AC}_{\mathrm{rec}}=1.4 \mathrm{kBq} / \mathrm{ml}, p=0.44\right)$ for $\mathrm{SCF}_{1.10}$ and $\mathrm{SCF}_{0.41}$, respectively, when compared with the NEMA acquisition protocol. The effect is exemplified by Fig. 1b.

Without recovery correction and independent of the total number of iterations, all reconstructions underestimate the known AC. For the largest examined sphere and with the phantom-specific $\mathrm{SCF}_{0.41}$, the $\mathrm{AC}_{\text {rec }}$ accuracy was in the range of -4.8 to $-13.8 \%$
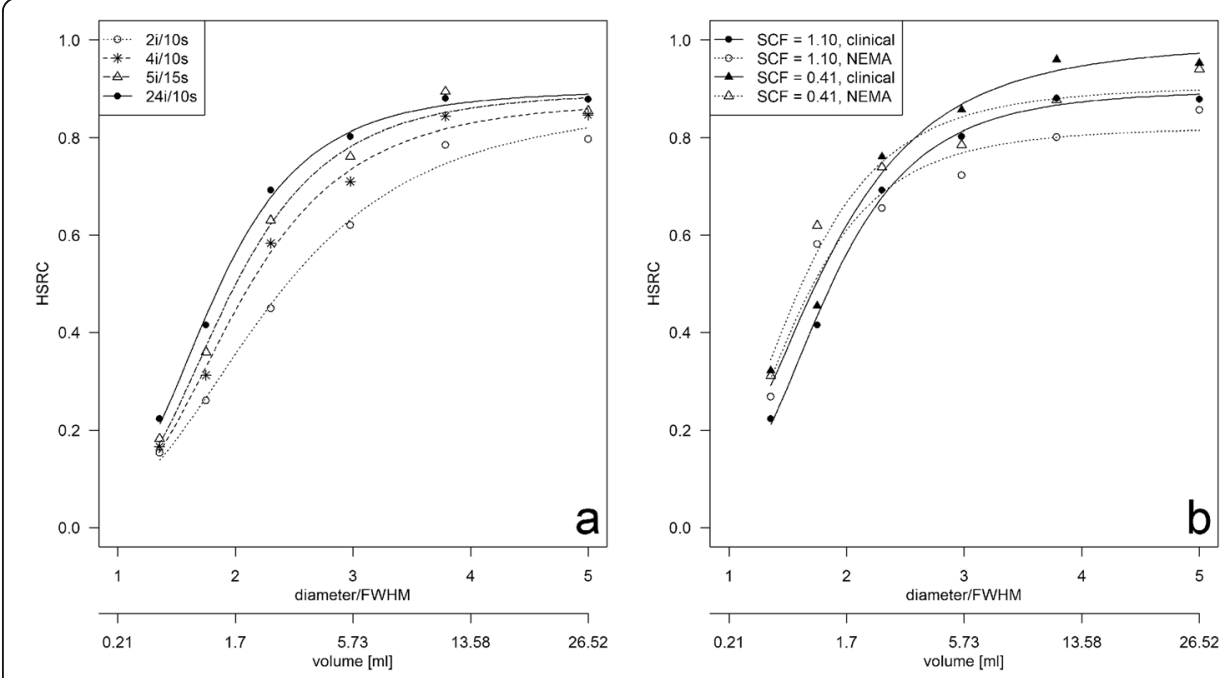

Fig. 1 Effect of the reconstruction setup on the hot spot recovery coefficients (HSRC) of the examined spheres. a HSRCs of the clinical acquisition protocol (60 projections, 20 s/projection) for different reconstruction setups ( $\mathrm{SCF}_{1.10}$ in combination with four different iteration sets). $\mathbf{b}$ HSRCs for a fixed set of iterations (24i/10s) demonstrating effects by the acquisition protocol (clinical or NEMA) and SCF (SCF $=1.10$ or 0.41 ). HSRC was plotted against sphere diameter normalized to FWHM at $100 \mathrm{~mm}$ with LEHR collimator $(\mathrm{FWHM}=7.4 \mathrm{~mm})$ and sphere volume 
(mean $=-8.4 \%,-7.1 \mathrm{kBq} / \mathrm{ml} ; p<0.0001)$ when compared with the known AC. All protocols with the default $\mathrm{SCF}_{1.10}$ underestimate the known $\mathrm{AC}$ in the largest sphere in the range of -12.2 to $-22.2 \%$ (mean $=-16.3 \%,-13.9 \mathrm{kBq} / \mathrm{ml} ; p<0.0001$ ). Generally, underestimation decreases with an increase in the total number of iterations (see supplementary data, Table 2).

\section{Background}

The $\mathrm{AC}_{\mathrm{rec}}$ of the background is higher than the known $\mathrm{AC}$ in the phantom for most of the examined reconstructions, and it is significantly affected by the SCF and the acquisition protocol (both, $p<0.0001$ ). We did not observe a significant effect of the total number of iterations on the $\mathrm{AC}_{\text {rec }}$ in the background $(p=1)$. Table 3 shows the results of a post hoc test for the background.

The smallest deviation of $\mathrm{AC}_{\mathrm{rec}}$ in the background from known $\mathrm{AC}$ was achieved by the clinical acquisition protocol with an $\mathrm{SCF}_{1.10}$. These reconstructions underestimate the known $\mathrm{AC}$ by a mean of $-1.8 \%$. The largest deviation from the true $\mathrm{AC}$ was determined for the NEMA acquisition protocol with $\mathrm{SCF}_{0.41}$ by a mean of $20.9 \%$.

\section{Qualitative results}

\section{Spheres}

The SNR was significantly affected by the sphere volume and by the reconstruction protocol used (both, $p<0.0001$, results by the Friedman test).

The results from ANOVA analysis showed, for each of the four largest examined sphere volumes, a significant influence by the number of total iterations (all, $p<$ $0.0001)$. The SCF $(p=0.01-0.08)$ and the acquisition protocol had no significant effect on the SNR (all, $p>0.06$ ). Table 2 exemplifies the results of an ANOVA post hoc test for the largest examined sphere. The results of the remaining sphere volumes are provided in the supplementary data (supplementary Table 1).

The effect of the total iterations and the sphere volume on the SNR was exemplified for the clinical acquisition protocol with the $\mathrm{SCF}_{1.10}$ (manufacturer recommendation) in Fig. 2a. The SNR in the images reconstructed by the four sets of iterations differed significantly $(p=0.0004)$, and there was an increase in the SNR with increasing VOI volumes and with a decreasing number of iterations. For example, the SNR for the

Table 3 Effect of examined parameters on background $A C_{\text {rec }}$ and $N$ in comparison to a reference protocol $^{\mathrm{a}}$

\begin{tabular}{|c|c|c|c|c|}
\hline $\begin{array}{l}\text { Parameter of } \\
\text { variation }\end{array}$ & $\begin{array}{l}\Delta \mathrm{AC}_{\mathrm{rec}} \\
(\mathrm{kBq} / \mathrm{ml})\end{array}$ & $p^{b}$ & $\begin{array}{l}\Delta N \\
(\%)\end{array}$ & $p^{b}$ \\
\hline SCF: 0.41 & +1.7 & $<0.0001$ & -6.0 & 0.02 \\
\hline Iteration set: $4 \mathrm{i} / 10 \mathrm{~s}$ & -0.1 & n.s. ${ }^{d}$ & +22.0 & $<0.0001$ \\
\hline Iteration set: $5 \mathrm{i} / 15 \mathrm{~s}$ & -0.1 & n.s. ${ }^{d}$ & +35.3 & $<0.0001$ \\
\hline Iteration set: $24 \mathrm{i} / 10 \mathrm{~s}$ & -0.2 & n.s. ${ }^{d}$ & +68.1 & $<0.0001$ \\
\hline Acquisition: NEMAc & +0.7 & $<0.0001$ & -4.1 & n.s. ${ }^{d}$ \\
\hline
\end{tabular}

${ }^{a}$ Clinical standard for diagnostic imaging and reconstruction (60 projections with $20 \mathrm{~s} /$ projection, $\mathrm{SCF}_{1.10}, 2 \mathrm{i} / 10 \mathrm{~s}$, Butterworth postfilter)

${ }^{\mathrm{b}}$ ANOVA post hoc test

${ }^{c}$ NEMA acquisition protocol (120 projections and $10 \mathrm{~s} /$ projection)

${ }^{\mathrm{d}}$ Not significant, tested by ANOVA

$S C F$ scatter weighting factor, $A C_{\text {rec }}$ mean reconstructed activity concentration, $N$ noise 

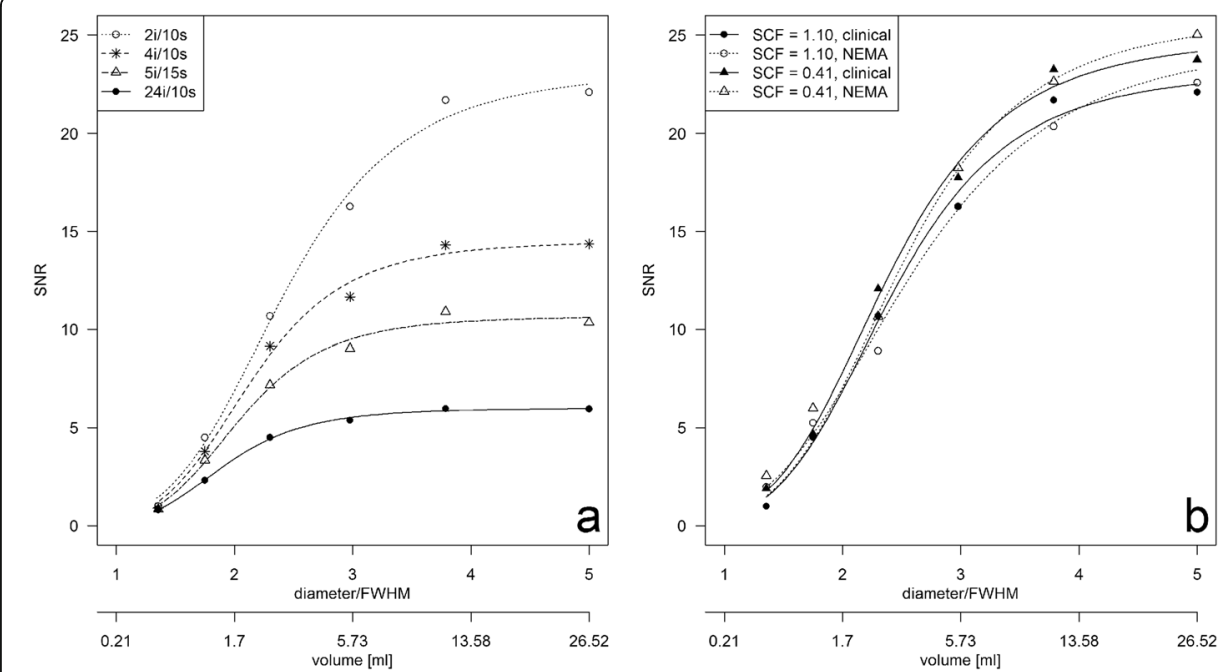

Fig. 2 Effect of the reconstruction setup on the signal-to-noise ratio (SNR) of the examined spheres. a SNR of the clinical acquisition protocol (60 projections, 20 s/projection) for different reconstruction setups (SCF 1.10 in combination with four different iteration sets). $\mathbf{b}$ SNR for a fixed set of iterations (2i/10s) demonstrating effects by the acquisition protocol (clinical or NEMA) and SCF (1.10 or 0.41). HSRC was plotted against sphere diameter normalized to FWHM at $100 \mathrm{~mm}$ with LEHR collimator (FWHM $=7.4 \mathrm{~mm}$ ) and sphere volume

smallest sphere is in the range from $0.8(24 \mathrm{i} / 10 \mathrm{~s})$ to $1.0(2 \mathrm{i} / 10 \mathrm{~s})$, while the SNR for the largest sphere varies depending on the total number of iterations from $6.0(24 \mathrm{i} / 10 \mathrm{~s})$ to $22.1(2 \mathrm{i} / 10 \mathrm{~s})$. Increasing the total number of iterations from $2 \mathrm{i} / 10 \mathrm{~s}$ to $4 \mathrm{i} / 10 \mathrm{~s}$ showed a significant decrease in median SNR by -3.9 over all spheres $(p=0.03)$. A further increase from $2 \mathrm{i} / 10 \mathrm{~s}$ to $5 \mathrm{i} / 15 \mathrm{~s}$ or $24 \mathrm{i} / 10$ s decreased the median SNR significantly by -5.9 $(p=0.03)$ or by $-8.5(p=0.03)$, respectively.

For a fixed set of iterations, for example $2 \mathrm{i} / 10 \mathrm{~s}$, the median SNR of the spheres increases for reconstructions with $\mathrm{SCF}_{0.41}$ in comparison to using an $\mathrm{SCF}_{1.10}$ significantly by 1.3 for the clinical and by 1.6 for the NEMA (both, $p=0.03$ ) acquisition protocol. Using the clinical acquisition protocol, the median SNR of the spheres decreased slightly by $-0.2(p=$ $0.84)$ and by $-0.3(p=0.69)$ for $\mathrm{SCF}_{1.10}$ and $\mathrm{SCF}_{0.41}$, respectively, when compared with the NEMA acquisition protocol. The effect is exemplified in Fig. 2b.

The highest SNR was observed for the diagnostic reconstruction setup (Table 1; 2i/ $10 \mathrm{~s}$ with postfiltering) and $\mathrm{SCF}_{0.41}$ (e.g., for the largest sphere; $\mathrm{SNR}_{\mathrm{NEMA}}=25.0, \mathrm{SNR}_{\text {cli- }}$ nical $=23.7)$. In contrast, using $24 \mathrm{i} / 10 \mathrm{~s}$, the $\mathrm{SNR}$ was reduced by $-80 \%\left(\mathrm{SNR}_{\mathrm{NEMA}}=\right.$ $5.1)$ and by $-74 \%\left(\mathrm{SNR}_{\text {clinical }}=6.2\right.$, both for the largest sphere $)$.

\section{Background}

Image noise $N$ in the background (Table 3 ) was significantly affected by the number of total iterations $(p<0.0001)$ and SCF $(p=0.01)$. The acquisition protocol had no significant effect on the SNR $(p=0.08)$.

General image quality of the quantitative reconstructions for the spheres and the background is shown in Fig. 3 for the clinical acquisition protocol and Fig. 4 for the NEMA acquisition protocol. Four of the six largest spheres in the phantom were clearly visible as spheres for all examined reconstructions. The non-active lung insert also 


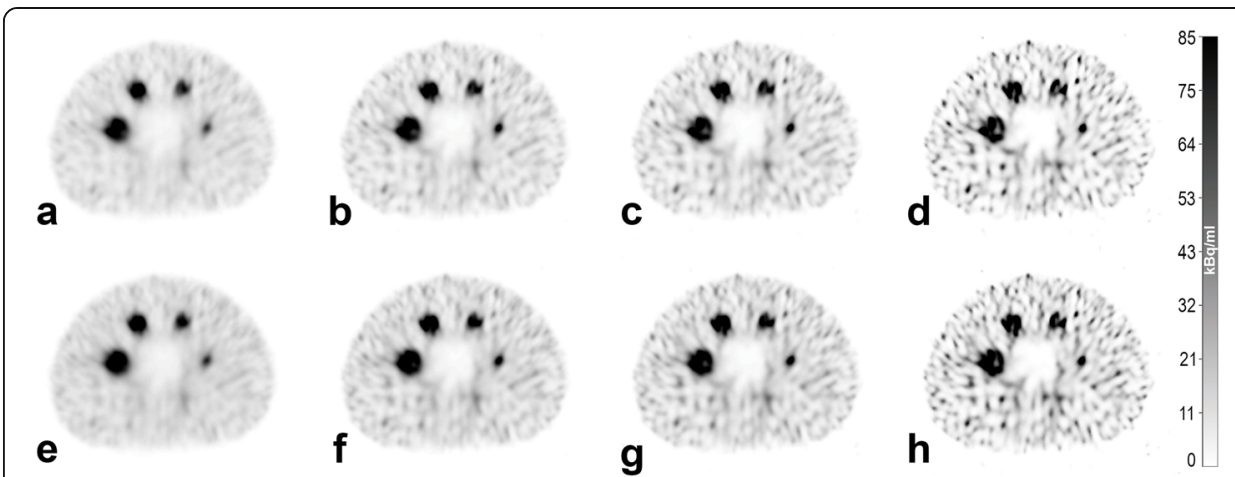

Fig. 3 SPECT images reconstructed from data acquired with the clinical acquisition protocol. Reconstruction was performed with 2i/10s (a and $\mathbf{e}$ ), 4i/10s (b and $\mathbf{f}$ ), 5i/15s (c and $\mathbf{g}$ ) and 24i/10s (d and $\mathbf{h}$ ). Scatter correction was performed with $\mathbf{a}-\mathbf{d}$ SCF $=1.10$ and $\mathbf{e}-\mathbf{h}$ SCF $=0.41$. SPECT images (a and $\mathbf{e}$ ) were reconstructed with postfiltering and represent the subjectively preferred image quality for reading. a represents our institutional standard for diagnostic imaging and reconstruction. All SPECT images have an identical window level and width

clearly differentiated from the background. The subjectively preferred image quality (MCK, HA, JW) is provided by the clinical acquisition protocol with $2 \mathrm{i} / 10$ s and postfiltering (Fig. 3a and e). There is no preference for a specific SCF. Here, $N$ ranges between $21 \%$ (Fig. 3e) and 25\% (Fig. 3a) for the background.

The highest noise in the background was provided by the reconstructions with $24 \mathrm{i} /$ 10s with $\mathrm{SCF}_{1.10}$ (Figs. 3/4d). Here, $N$ ranges between 106\% (Fig. 3d) and 116\% (Fig. $4 d$ ). The results for all examined protocols and additional descriptive statistics are provided in the supplementary data (supplementary Tables 2 and 3).

\section{Discussion}

A common clinically used qSPECT reconstruction algorithm was evaluated regarding quantitative accuracy and image quality using a standard phantom geometry. SPECT data acquired by two different acquisition protocols, a clinical protocol and a NEMAoriented scan protocol, were reconstructed by iterative algorithms with different parametrizations (e.g., variations in the number of iterations and different factors for

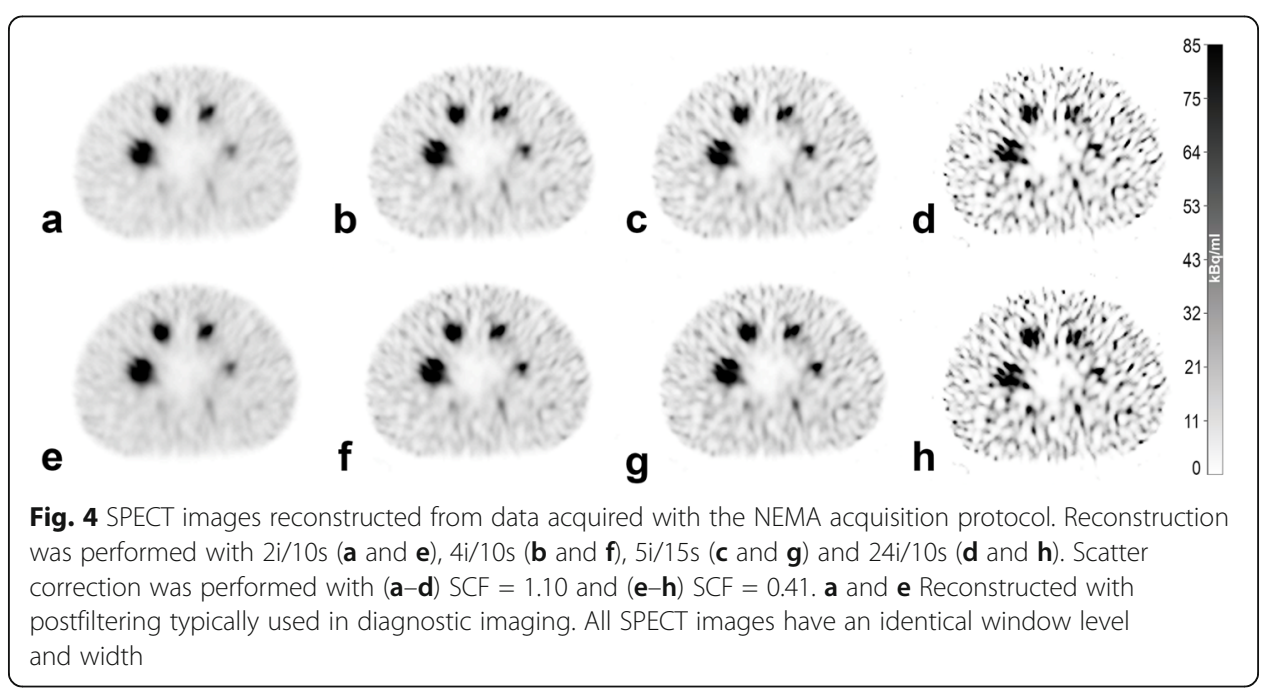


scatter correction). The two acquisition protocols were chosen to analyse the effect introduced by different numbers of projections for equivalent total examination times. Reconstructed data were evaluated regarding the effect of scatter correction and of the total number of iterations on the determined activity concentration. In addition, we compared the results of the quantitative reconstruction setups with a reconstruction setup used as a standard for diagnostic imaging in routine clinical practice. The phantom setup was chosen in concordance with activity concentrations typical for diagnostic workflows using ${ }^{99 \mathrm{~m}}$ Tc-labelled radiopharmaceuticals.

Various setups for activity concentrations and activity ratios (e.g., sphere-tobackground) are reported in the literature. Livieratos et al. [28] performed phantom measurements representing a bone scan-oriented setup with an activity concentration ratio of 4.8:1 (sphere $=240 \mathrm{kBq} / \mathrm{ml}$ and background $=50 \mathrm{kBq} / \mathrm{ml}$ ). Collarino et al. [25] performed phantom measurements based on breast cancer imaging with four sets of activity concentration ratios and different background activity concentrations (e.g., sphere-to-background ratio of 9.7:1 and background $=3.6 \mathrm{kBq} / \mathrm{ml}$ ). A showcase from this work reveals tumour-to-background ratios of 10:1 and 8:1 for the early (5 min p.i.) and delayed (90 min p.i.) acquisition. However, since our approach is not limited to a particular type of examination and the focus is primarily on quantitative analysis, we chose other activity concentrations. We assume that as long as the dead time is not exceeded, the investigated activity concentrations are of secondary importance compared with the object size with respect to the spatial resolution of the camera.

In our phantom setup, the default $\mathrm{SCF}_{1.10}$ in image reconstruction overestimates the portion of scattered photons within the photopeak window by a factor of 2.7. This result resulted in a significantly reduced $\mathrm{AC}_{\mathrm{rec}}$ compared with the known $\mathrm{AC}$ in the phantom. Furthermore, qualitative parameters (e.g., SNR) were artificially affected because of the reduced count statistics. Thus, scatter correction with the objectoptimized $\mathrm{SCF}_{0.41}$ resulted in an increased $\mathrm{AC}_{\mathrm{rec}}$ closer to the real $\mathrm{AC}$. Additionally, qualitative results were improved compared to results with default $\mathrm{SCF}_{1.10}$. However, both SCFs overestimate the known AC of the background. This finding has to be discussed regarding the methodology for estimating the SCF by using a small activity filled sphere in an inactive homogenous-filled water phantom (see supplementary information) [23].

Another objective for qSPECT was the parametrization of the quantitative reconstruction algorithm (e.g., number of iterations and subsets). We examined four different sets of iterations for the iterative OSEM algorithm. As known from PET imaging, with an increasing number of iterations, $\mathrm{AC}_{\mathrm{rec}}$ increased and converged to the known $\mathrm{AC}$ [29]. However, a residual offset (underestimation of the AC) was observed. In parallel, image noise increased with an increasing number of iterations. On the other hand, the number of iterations showed no effect on the background AC. For a homogeneous, large object (e.g., the background VOI), it can be assumed that the reconstruction algorithm converges after a few iterations. It has to be hypothesized that the lowest examined number of total iterations (2i/10s) was sufficient to show convergence. Therefore, an effect introduced by the number of iterations on background $\mathrm{AC}$ was not observed.

Finally, the acquisition protocol (number of projections and duration of each projection) demonstrated a minor impact on the quantitative results for the spheres, in contrast to SCF or total number of iterations. Furthermore, there was no significant 
influence of the acquisition protocol on the estimated image quality parameters. In contrast, the acquisition protocol has an influence on the background. For the background, the best quantitative results were obtained with the clinical protocol, and the best qualitative results were obtained with the NEMA protocol. Using the NEMA protocol, the AC reconstructed in the background was overestimated compared with the known AC. At the same time, the standard deviations of the ACs are smaller with the NEMA protocol than with the clinical protocol, resulting in a reduction in image noise. Here, we concluded that the acquisition parameters, such as the number of projections and duration of projection time, are less relevant in volumes with high-count statistics (e.g., in the spheres) compared with regions with low-count statistics (e.g., in the background).

In summary, the best quantitative results were achieved with the object-specific $\mathrm{SCF}_{0.41}$ and a reconstruction set with 24 iterations and 10 subsets without postfiltering. In contrast, the best qualitative results were achieved with $\mathrm{SCF}_{0.41}$ with 2 iterations and 10 subsets and with postfiltering. In this parametrization, however, for diagnostic reading, the clinical acquisition protocol (regardless of SCF) was subjectively preferred.

In addition to the effects examined, further corrections have to be considered in qSPECT imaging (e.g., for photon attenuation, patient motion, dead time, radioactive decay and postfiltering of data) [4, 7-12]. These effects have not been analysed here.

In our study, photon attenuation was determined from low-dose CT data. Here, optimization of low-dose CT (e.g., the use of iterative reconstruction techniques) was demonstrated to be an innovative approach [30-32]. The influence/correction of dead time is not appropriate for the examined activity concentration typically observed in diagnostic applications but should be considered in the imaging of therapy nuclides (e.g., internal radionuclide therapy with ${ }^{177} \mathrm{Lu}$ ) using high activities [33].

One limitation in our reconstruction process is the use of a mean planar sensitivity value for the conversion of SPECT counts to an activity concentration. The software used only a single system sensitivity for both detector systems, even though the sensitivities of the single detectors slightly differed for the examined radionuclide. The sensitivity is determined only from planar imaging; thus, the sensitivity depends on the object-related properties (e.g., dimension and resulting scattering). A potential approach to overcome specific methodological limitations is the use of a three-dimensional sensitivity value by measuring a homogeneously filled phantom with known activity [9].

Furthermore, our approach is limited by the estimation of the object-specific SCF, which in turn depends on density composition, object shape, VOI volume and activity concentration [17]. Here, the reconstruction can perhaps be improved by Monte Carlobased individual scatter correction [15, 34]. In the comparison between the Monte Carlo-based scatter correction and a correction with additional energy windows, Gils et al. [35] demonstrated for the NEMA phantom that the results of the reconstructed activity in the spheres and in the background differ by less than $10 \%$ for ${ }^{131} \mathrm{I}$. However, the portion of scattered photons within the photopeak of ${ }^{131}$ I may be different from ${ }^{99 \mathrm{~m}} \mathrm{Tc}$, and an improvement may nevertheless be expected. Xiao et al. [36] showed for different phantoms that Monte Carlo-based scatter correction for ${ }^{99} \mathrm{~m}$ Tc in cardiac perfusion SPECT is superior to conventional window-based scatter correction. With identical noise levels, the contrast of the Monte Carlo-based scatter correction is 10 to $20 \%$ higher. 
Furthermore, SPECT imaging and quantitative analysis are always influenced by other effects (e.g., resolution recovery). In our setup, only the four largest spheres (volume $\geq$ $2.5 \mathrm{ml}$ ) could be clearly separated from the background in the reconstructed SPECT data. However, segmentation was performed CT-based to prevent an additional bias by SPECT data-oriented delineation of contours. Furthermore, the whole analysis was performed for the largest sphere only to limit the influence of the partial volume effect on the primary analysis of HSRCs and SNR (e.g., caused by definitions of parameters, supplementary data showing results from all spheres). Nevertheless, depending on the spatial resolution of the imaging system, recovery correction is recommended [26].

In the future, the utilization of qSPECT imaging will become more important in routine clinical practice. The predicted use of this method is especially true for dosimetry in novel treatments (e.g., in radionuclide therapies) [5, 6]. Diagnostics will also benefit from qSPECT imaging, as it improves intraindividual comparability and even allows direct comparison of reconstructions of different SPECT/CT devices [37-40]. Further developments might focus on optimizing SPECT images for CT metal artefacts, such as those that occur for medical devices (e.g., postsurgery prosthesis management [41]).

Therefore, we recommend performing reconstruction for diagnostic imaging and auxiliary reconstruction optimized for quantitative analysis. From our phantom study, reconstruction with $24 \mathrm{i} / 10 \mathrm{~s}$ is the best choice for quantitative reconstruction.

\section{Conclusion}

Quantitative SPECT imaging is feasible with the used (commercially available) reconstruction algorithm and hybrid SPECT/CT, and its consistent implementation in diagnostics may provide perspectives for quantification in routine clinical practice. When combining quantitative analysis and diagnostic imaging, we recommend using two different reconstruction protocols with task-specific optimized setups (quantitative vs qualitative reconstruction). Furthermore, individual scatter correction significantly improves both quantitative and qualitative results. Accepting factory settings may lead to errors and should always be critically questioned. The conversion of measured counts into quantitative measured quantities $(\mathrm{Bq} / \mathrm{ml})$ by the used quantitative factor leads to acceptable accuracies (less than $10 \%$ deviation from the true activity concentration) for spheres up to $11.5 \mathrm{ml}$ in volume if appropriate image data acquisition and reconstruction are used.

\section{Abbreviations}

2i/10s: Two iterations and 10 subsets; 4i/10s: Four iterations and 10 subsets; $5 \mathrm{i} / 15 \mathrm{~s}$ : Five iterations and 15 subsets; $24 \mathrm{i} /$ 10s: Twenty-four iterations and 10 subsets; ${ }^{99 \mathrm{~m}} \mathrm{Tc}$ : Technetium-99m; ${ }^{177} \mathrm{Lu}$ : Lutetium-177; AC: Activity concentration; CT: Computer tomography; FWHM: Full width at half maximum; HSRC: Hot spot recovery coefficient; LEHR: Lowenergy, high resolution; N: Image noise; NEMA IEC: National Electrical Manufacturers Association International Electrotechnical Commission; OSEM: Ordered subset expectation maximization; PET: Positron emission tomography; qSPECT: Quantitative SPECT; SCF: Scatter weighting factor; SD: Standard deviation; SNR: Signal-to-noise ratio; SPEC

T: Single photon emission computed tomography; VOI: Volume of interest

\section{Supplementary Information}

The online version contains supplementary material available at https://doi.org/10.1186/s40658-021-00405-3.

Additional file 1. Determination of the weighting factor for scatter correction (SCF)

Additional file 2: Table S1 Effect of examined parameter on $A_{r e c}, H S R C$ and SNR. Results are exemplified for all sphere volumes in comparison to a reference protocol

Additional file 3: Table S2 AC $_{\text {rec }}, H S R C$ and SNR from all sphere volumes of the phantom ${ }^{a}$ for all examined acquisition and reconstruction protocols 
Additional file 4: Table $\mathbf{S 3} \mathrm{AC}_{\mathrm{rec}}$ and noise from background ${ }^{\mathrm{a}}$ for all examined acquisition and reconstruction protocols

\section{Acknowledgements}

Not applicable

\section{Authors' contributions}

The conceptualization of this study was carried out by HA, OSG and DK. Methodology was developed by DK, HW and OSG, and the measurements were performed by DK and HW. The analysis of the data, statistics and visualization was done by DK, JW and SB. The original draft was written by DK. The draft was reviewed and edited by all authors. The supenvision was done by OSG. Funds and resources were provided by OSG, MCK, HA and MP. The authors read and approved the final manuscript.

\section{Funding}

DK, HA and OSG received a research grant by GE Healthcare. This publication was funded by the German Research Foundation (DFG) and the University of Magdeburg in the funding program Open Access Publishing. The work of this paper is partly funded by the Federal Ministry of Education and Research within the Research Campus STIMULATE under grant number 13GW0473A. Open Access funding enabled and organized by Projekt DEAL.

\section{Availability of data and materials}

SPECT and CT raw datasets are available from the corresponding author on reasonable request. All data analysed during this study are included in this published article and its supplementary information files.

\section{Declarations}

\section{Ethics approval and consent to participate}

Not applicable

\section{Consent for publication}

Not applicable

\section{Competing interests}

DK, HA and OSG received an unrestricted research grant provided by GE Healthcare. All other authors declare that they have no competing interests.

\section{Author details}

${ }^{1}$ Department of Radiology and Nuclear Medicine, University Hospital Magdeburg, Magdeburg, Germany. ${ }^{2}$ Department of Nuclear Medicine, Charité - Universitätsmedizin Berlin, Corporate Member of Freie Universität Berlin, Humboldt-Universität zu Berlin, and Berlin Institute of Health, Berlin, Germany. ${ }^{3}$ Research Campus STIMULATE, Otto-von-Guericke University, Magdeburg, Germany.

Received: 26 March 2021 Accepted: 13 July 2021

Published online: 30 July 2021

\section{References}

1. Boellaard R, Delgado-Bolton R, Oyen WJG, Giammarile F, Tatsch K, Eschner W, et al. FDG PET/CT: EANM procedure guidelines for tumour imaging: version 2.0. EJNMMI. 2015. https://doi.org/10.1007/s00259-014-2961-x.

2. Quak E, Le Roux PY, Hofman MS, Robin P, Bourhis D, Callahan J, et al. Harmonizing FDG PET quantification while maintaining optimal lesion detection: prospective multicentre validation in 517 oncology patients. EJNMMI. 2015;42(13): 2072-82. https://doi.org/10.1007/s00259-015-3128-0.

3. Dickson J, Ross J, Vöö S. Quantitative SPECT: the time is now. EJNMMI Phys. 2019. https://doi.org/10.1186/s40658-019-0241-3.

4. Bailey DL, Willowson KP. An evidence-based review of quantitative SPECT imaging and potential clinical applications. J Nucl Med. 2013;54(1):83-9. https://doi.org/10.2967/jnumed.112.111476.

5. Ljungberg M, Celler A, Konijnenberg MW, Eckerman KF, Dewaraja YK, Sjögreen-Gleisner K, et al. MIRD Pamphlet No. 26 joint EANM/MIRD guidelines for quantitative ${ }^{177}$ Lu SPECT applied for dosimetry of radiopharmaceutical therapy. J NuCl Med. 2016. https://doi.org/10.2967/jnumed.115.159012.

6. Delker A, Fendler WP, Kratochwil C, Brunegraf A, Gosewisch A, Gildehaus FJ, et al. Dosimetry for (177)Lu-DKFZ-PSMA-617: a new radiopharmaceutical for the treatment of metastatic prostate cancer. ENMMI. 2016:43(1):42-51. https:/doi.org/10.1007/s00259-015-3174-7.

7. Rosenthal MS, Cullom J, Hawkins W, Moore SC, Tsui BM, Yester M. Quantitative SPECT imaging: a review and recommendations by the focus committee of the Society of Nuclear Medicine Computer and Instrumentation Council. J Nucl Med. 1995;36(8):1489-513.

8. Zeintl J, Vija AH, Yahil A, Hornegger J, Kuwert T. Quantitative accuracy of clinical ${ }^{99 m}$ TC SPECT/CT using ordered-subset expectation maximization with 3-dimensional resolution recovery, attenuation, and scatter correction. J Nucl Med. 2010; 51(6):921-8. https://doi.org/10.2967/jnumed.109.071571.

9. Ritt P, Vija H, Hornegger J, Kuwert T. Absolute quantification in SPECT. EJNMMI. 2011;38(S1):69-77. https://doi.org/10.1 007/s00259-011-1770-8

10. Dewaraja YK, Frey EC, Sgouros G, Brill AB, Roberson P, Zanzonico PB, et al. MIRD Pamphlet No. 23: quantitative SPECT for patientspecific 3-dimensional dosimetry in internal radionuclide therapy. J Nucl Med. 2012. https://doi.org/10.2967/jnumed.111.100123.

11. Bailey DL, Willowson KP. Quantitative SPECT/CT: SPECT joins PET as a quantitative imaging modality. EJNMMI. 2014:53(8): 1310-25. https://doi.org/10.2967/jnumed.111.100123. 
12. Sanders JC, Kuwert T, Hornegger J, Ritt P. Quantitative SPECT/CT imaging of ${ }^{177}$ Lu with in vivo validation in patients undergoing peptide receptor radionuclide therapy. Mol Imaging Biol. 2015;53(8):1310-25. https://doi.org/10.2967/jnumed.111.100123.

13. Zaidi H, Hasegawa B. Determination of the attenuation map in emission tomography. J Nucl Med. 2003;44(2):291-315.

14. Willowson K, Bailey DL, Baldock C. Quantitative SPECT reconstruction using CT-derived corrections. Phys Med Biol. 2008; 53(12):3099-112. https://doi.org/10.2967/jnumed.111.100123.

15. [Hutton 2011] Hutton BF, Buvat I, Beekman FJ. Review and current status of SPECT scatter correction. Phys Med Biol 2011; doi:https://doi.org/10.1088/0031-9155/56/14/R01, 56, 14, R85, R112

16. Jaszczak RJ, Greer KL, Floyd CE Jr, Harris CC, Coleman RE. Improved SPECT quantification using compensation for scattered photon. J Nucl Med. 1984;25(8):893-900.

17. Koral KF, Swailem FM, Buchbinder S, Clinthorne NH, Rogers WL, Tsui BM. SPECT dual-energy-window Compton correction: scatter multiplier required for quantification. J Nucl Med. 1990;31(1):90-8.

18. Ogawa K, Harata Y, Ichihara T, Kubo A, Hashimoto S. A practical method for position-dependent Compton-scatter correction in single photon emission CT. IEEE Trans Med Imaging. 1991;10(3):408-12. https://doi.org/10.1109/42.97591.

19. de Nijs R, Lagerburg V, Klausen TL, Holm S. Improving quantitative dosimetry in ${ }^{177}$ Lu-DOTATATE SPECT by energy windowbased scatter corrections. Nucl Med Commun. 2014;35(5):522-33. https:/doi.org/10.1097/MNM.0000000000000079.

20. National Electrical Manufacturers Association. Performance measurements of positron emission tomographs (PET). NEMA Standards Publication NU 2-2018. Virginia: National Electrical Manufacturers Association; 2018.

21. National Electrical Manufacturers Association. Performance measurements of gamma cameras. NEMA Standards Publication NU 1-2018. Virginia: National Electrical Manufacturers Association; 2018.

22. GE Healthcare. NM quantification, Q.Metrix for SPECT/CT package: White Paper; 2017.

23. de Nijs R, Holm S, Thomsen G, Ziebell M, Svarer C. Experimental determination of the weighting factor for the energy window subtraction-based downscatter correction for ${ }^{123}$ in brain SPECT studies. J Med Phys. 2010;35(4):215-22. https://doi.org/10.4103/0971-6203.71765.

24. Ichihara T, Ogawa K, Motomura N, Kubo A, Hashimoto S. Compton scatter compensation using the triple-energy window method for single- and dual-isotope SPECT. J Nucl Med. 1993;34(12):2216-21.

25. Collarino A, Pereira Arias-Bouda LM, Valdés Olmos RA, van der Tol P, Dibbets-Schneider P, de Geus-Oei LF, et al. Experimental validation of absolute SPECT/CT quantification for response monitoring in breast cancer. Med Phys. 2018, 45(5):2143-53. https://doi.org/10.1002/mp.12880.

26. Geworski L, Knoop BO, Cabrejas ML, Knapp WH, Munz DL. Recovery correction for quantification in emission tomography: a feasibility study. Eur J Nucl Med. 2000;27(2):161-9. https://doi.org/10.1007/s002590050022.

27. Noori-Asl M, Sadremomtaz A, Bitarafan-Rajabi A. Evaluation of three scatter correction methods based on estimation of photopeak scatter spectrum in SPECT imaging: a simulation study. Phys Med. 2014;30(8):947-53. https://doi.org/10.1016/j.jemp.2014.05.008.

28. Livieratos L, Mohan H, Gnanasegaran G, Fogelman I. Comparison of 10 versus 20 min SPECT ${ }^{99 m}$ Tc-MDP bone scans: use of 3D-OSEM image reconstruction with distance-dependent resolution modelling. Nucl Med Commun. 2010;31(12): 1045-53. https://doi.org/10.1097/MNM.0b013e32833fba6e.

29. Jaskowiak CJ, Bianco JA, Perlman SB, Fine JP. Influence of reconstruction iterations on 18-F-FDG PET/CT standardized uptake values. J Nucl Med. 2005:46(3):424-8.

30. Grosser OS, Kupitz D, Ruf J, Czuczwara D, Steffen IG, Furth C, et al. Optimization of SPECT-CT hybrid imaging using iterative image reconstruction for low-dose CT: a phantom study. PLoS One. 2015;10(9):e0138658. https://doi.org/10.13 71/journal.pone.0138658.

31. Sibille L, Chambert B, Alonso S, Barrau C, D'Estanque E, Tabaa Y, et al. Impact of the adaptive statistical iterative reconstruction technique on radiation dose and image quality in bone SPECT/CT. J Nucl Med. 2016;57(7):1091-5. https://doi.org/10.2967/jnumed.115.164772.

32. Grosser OS, Ruf J, Kupitz D, Czuczwara D, Loewenthal D, Thormann M, et al. Iterative CT reconstruction in abdominal low-dose CT used for hybrid SPECT-CT applications: effect on image quality, image noise, detectability, and reader's confidence. Acta Radiol Open. 2019;8(6):205846011985626. https://doi.org/10.1177/2058460119856266.

33. Frezza A, Desport C, Uribe C, Zhao W, Celler A, Després $P$, et al. Comprehensive SPECT/CT system characterization and calibration for 177Lu quantitative SPECT (QSPECT) with dead-time correction. EJNMMI Phys. 2020;7(1):10. https://doi. org/10.1186/s40658-020-0275-6.

34. Kangasmaa TS, Constable C, Sohlberg AO. Evaluation of quantitative ${ }^{123}$ and ${ }^{131}$ I SPECT with Monte Carlo-based downscatter compensation. Nucl Med Commun. 2018;39(12):1097-102. https://doi.org/10.1097/MNM.0000000000000920.

35. van Gils CAJ, Beijst C, van Rooij R, de Jong HWAM. Impact of reconstruction parameters on quantitative I-131 SPECT. Phys Med Biol. 2016. https://doi.org/10.1088/0031-9155/61/14/5166.

36. Xiao J, de Wit TC, Staelens SG, Beekman FJ. Evaluation of 3D Monte Carlo-based scatter correction for ${ }^{99 \mathrm{~m}} \mathrm{Tc}$ cardiac perfusion SPECT. J Nucl Med. 2006;48(4):637-44. https://doi.org/10.2967/jnumed.106.037259.

37. Peters SMB, Werf NR, Segbers M, Velden FHP, Wierts R, Blokland KJAK, et al. Towards standardization of absolute SPECT/CT quantification: a multi-center and multi-vendor phantom study. ENMMMI Phys. 2019;6(1):29. https://doi.org/10.1186/s40658-019-0268-5.

38. Kennedy JA, Lugassi R, Gill R, Keidar Z. Digital solid-state SPECT/CT quantitation of absolute ${ }^{177}$ Lu-radiotracer concentration: in vivo/in vitro validation. J Nucl Med. 2020;61(9):1381-7. https://doi.org/10.2967/jnumed.119.239277.

39. Bellevre D, Bailliez A, Delelis F, Blaire T, Agostini D, Mouquet F, et al. Quantitation of myocardial ${ }^{99 m}$ Tc-HMDP uptake with new SPECT/CT cadmium zinc telluride (CZT) camera in patients with transthyretin-related cardiac amyloidosis: Ready for clinical use? J Nucl Cardiol. 2020. https://doi.org/10.1007/s12350-020-02274-2.

40. Dittmann $H$, Kaltenbach $S$, Weissinger M, Fiz F, Martus P, Pritzkow M, et al. The prognostic value of quantitative bone SPECT/CT before ${ }^{223}$ Ra treatment in metastatic castration-resistant prostate cancer. J Nucl Med. 2021;62(1):48-54. https:// doi.org/10.2967/jnumed.119.240408.

41. Berth A, März V, Wissel H, Awiszus F, Amthauer H, Lohmann CH. SPECT/CT demonstrates the osseointegrative response of a stemless shoulder prosthesis. J Shoulder Elb Surg. 2016;25(4):e96-e103. https://doi.org/10.1016/j.jse.2015.09.009.

\section{Publisher's Note}

Springer Nature remains neutral with regard to jurisdictional claims in published maps and institutional affiliations. 